\title{
Operation Naloxone: Overdose Prevention Service Learning for Student Pharmacists
}

Lucas G. Hill, PharmD [Corresponding author]

Clinical Assistant Professor

The University of Texas at Austin College of Pharmacy

2409 University Avenue

A1910, PHR 2.222G

Austin, TX 78712

Phone 512-232-7832

lucas.hill@austin.utexas.edu

John Patrick Sanchez, PharmD

4606 Green Cottage Lane

Missouri City, TX 77459

Phone 281-854-8826

jpsanchez94@utexas.edu

S. Andrea Laguado, PharmD

3500 Greystone Drive

Apartment 165

Austin, TX 78731

Phone 817-734-6141

a.laguado@utexas.edu

Kenneth A. Lawson, PhD

Professor

The University of Texas at Austin College of Pharmacy

2409 University Avenue

A1930, PHR 3.209C

Austin, TX 78712

Phone 512-471-5609

ken.lawson@austin.utexas.edu

\section{Abstract}

Background \& Purpose A service learning program for student pharmacists was developed to train other university students to respond effectively to opioid overdoses with naloxone. Assessments were analyzed to determine the effect of program participation on student pharmacists' overdose-related knowledge retention and harm reduction attitudes.

Educational Activity \& Setting Student pharmacists were invited to attend a 90-minute train-the-trainer seminar to obtain foundational knowledge regarding opioid overdose risk, symptoms, and response. Attendees were eligible to participate in a series of 10 community outreach events to educate university students. These two-hour events included a 30-minute team huddle, 60-minute workshop, and 30-minute team debrief. Student pharmacists were asked to complete a follow-up assessment to evaluate knowledge retention and harm reduction attitudes. 


\section{DO NOT REFORMAT}

Findings Responses from students who participated in community outreach events (intervention) were compared to those who only attended the train-the-trainer seminar (control). A total of 116 subjects attended a train-the-trainer seminar and 94 completed the follow-up assessment. Thirty-six subjects voluntarily participated in at least one community outreach event while 58 did not participate. The intervention group demonstrated superior knowledge retention compared to the control group $(p<0.001)$. Cumulative harm reduction attitudes did not differ between groups $(p=0.89)$. The intervention group exhibited more positive attitudes regarding naloxone access for individuals who use illicit opioids $(p=0.015)$.

Summary The Operation Naloxone service learning program enabled student pharmacists to engage with their community while reinforcing overdose-related knowledge. Student pharmacists exhibited progressive attitudes regarding harm reduction interventions.

Keywords: Service learning, harm reduction, overdose prevention, opioids, naloxone

Disclosures: Dr. Hill reports receiving free medication from Kaléo Pharma and Adapt Pharma to support overdose prevention activities. None of the other authors have any relevant financial disclosures or conflicts of interest to report. 


\section{Operation Naloxone: Overdose Prevention Service Learning for Student Pharmacists}

\section{Abstract}

Background \& Purpose A service learning program for student pharmacists was developed to train other university students to respond effectively to opioid overdoses with naloxone. Assessments were analyzed to determine the effect of program participation on student pharmacists' overdose-related knowledge retention and harm reduction attitudes.

Educational Activity \& Setting Student pharmacists were invited to attend a 90-minute train-the-trainer seminar to obtain foundational knowledge regarding opioid overdose risk, symptoms, and response. Attendees were eligible to participate in a series of 10 community outreach events to educate university students. These two-hour events included a 30-minute team huddle, 60-minute workshop, and 30-minute team debrief. Student pharmacists were asked to complete a follow-up assessment to evaluate knowledge retention and harm reduction attitudes.

Findings Responses from students who participated in community outreach events (intervention) were compared to those who only attended the train-the-trainer seminar (control). A total of 116 subjects attended a train-the-trainer seminar and 94 completed the follow-up assessment. Thirty-six subjects voluntarily participated in at least one community outreach event while 58 did not participate. The intervention group demonstrated superior knowledge retention compared to the control group $(p<0.001)$. Cumulative harm reduction attitudes did not differ between groups $(p=0.89)$. The intervention group exhibited more positive attitudes regarding naloxone access for individuals who use illicit opioids $(p=0.015)$.

Summary The Operation Naloxone service learning program enabled student pharmacists to engage with their community while reinforcing overdose-related knowledge. Student pharmacists exhibited progressive attitudes regarding harm reduction interventions.

Keywords: Service learning, harm reduction, overdose prevention, opioids, naloxone 
Disclosures: Dr. Hill reports receiving free medication from Kaléo Pharma and Adapt Pharma to support overdose prevention activities. None of the other authors have any relevant financial disclosures or conflicts of interest to report.

\section{Background \& Purpose}

The Centers for Disease Control report that 52,404 Americans died from drug overdoses in 2015, and opioids contributed to $63 \%$ of these deaths. ${ }^{1}$ Naloxone, a potent mu-opioid receptor antagonist, is the only drug approved by the Food and Drug Administration for reversal of an acute opioid overdose. Several naloxone formulations are available with significant variations in cost and ease-of-use. ${ }^{2}$ Harm reduction organizations in the United States have been working for decades to expand public access to naloxone. From 1996-2010, these programs distributed naloxone to 152,283 individuals with 26,463 overdose reversals reported. ${ }^{3}$ Based on the success of this approach, at least 47 states have enacted naloxone access laws that allow pharmacists to dispense naloxone via standing order or another mechanism without requiring a medical encounter. ${ }^{4}$ Recognizing a growing role for pharmacists in opioid overdose prevention, the American Association of Colleges of Pharmacy issued a pledge to provide pertinent education to student pharmacists signed by 96 member institutions as of July 2017.

Service learning is not mentioned in standards issued by the Accreditation Council for Pharmacy Education, though an associated guidance document references the pedagogy as an avenue to "broaden student experiences" that may qualify as introductory pharmacy practice experience (IPPE). ${ }^{5-6}$ Recent studies of student pharmacists completing service learning experiences have demonstrated positive effects on cultural competence, ${ }^{7-8}$ communication skills, ${ }^{9-11}$ and self-efficacy. ${ }^{12-13} \mathrm{~A}$ service learning course at the University of Toronto connected students with local organizations to engage in harm reduction programs, and all fifteen students reported their views on drug misuse programs had changed as a result. ${ }^{14}$ Four published manuscripts have described overdose prevention training programs for and from student pharmacists, with some reporting positive impacts on overdose-related knowledge. ${ }^{15-18}$ No available studies have evaluated the impact of overdose prevention service learning on student pharmacists' overdose-related knowledge or harm reduction attitudes. 
The University of Texas at Austin has become a leader in institutional opioid overdose prevention by stocking naloxone rescue kits in residence halls and providing them to university police. College of Pharmacy faculty have been engaged in the process and collaborated with faculty from the School of Social Work and the Texas Overdose Naloxone Initiative to provide overdose response training to every resident advisor and police officer on campus. The objective of Operation Naloxone was to build on that foundation by developing and implementing an overdose prevention service learning program, within which student pharmacists would train other university students to identify and respond to suspected opioid overdoses with naloxone. We hypothesized that program participation would result in superior overdose-related knowledge retention and more positive attitudes regarding harm reduction interventions among student pharmacists.

\section{Educational Activity \& Setting}

Preliminary details of this program were previously described in a report from the American Association of Colleges of Pharmacy's advocacy committee. ${ }^{19}$ The faculty director engaged the presidents of two student pharmacist organizations, the Student College of Clinical Pharmacy and the Student Pharmacist Recovery Network, to co-develop Operation Naloxone as student directors. Learning materials were developed for a faculty-led, 90-minute, train-the-trainer seminar to prepare student pharmacists for future community outreach events. The seminar included a 60 -minute slide presentation to convey foundational knowledge, including opioid overdose epidemiology, physiology, risk factors, symptoms, naloxone formulations, and evidence-based response. The slide presentation was followed by a nine-minute video demonstrating proper administration technique for every available formulation of naloxone. Finally, a variety of questions likely to be encountered at community outreach events were discussed and evidence-based answers were reviewed. A faculty member with previous training as a paramedic provided insight and collaborated to record administration videos. A harm reduction partner secured donations of a branded intranasal naloxone product to disseminate during community outreach events. 
The student directors coordinated scheduling of three train-the-trainer seminars throughout the academic year (September 2016-February 2017) and invited student pharmacists from professional years 1-3 to attend via email and Facebook. Following these initial trainings, they scheduled 10 community outreach events for various groups of university students, primarily at off-campus student housing cooperatives (September 2016-April 2017). Each community outreach event encompassed two hours and qualified for required IPPE credit that is built into every semester of the College of Pharmacy curriculum. The events began with a 30-minute team huddle to review presentation content and setup audio/visual equipment. This was followed by a 60 -minute presentation adapted from the seminar content into a 30-minute lecture and a 30-minute case-based discussion in small groups. The lectures were delivered by professional year three (P3) student pharmacists given their higher level of experience with professional presentations. Small groups were facilitated by professional year one and two (P1-2) student pharmacists with supervision from P3 student pharmacists. The service learning experience ended with a 30-minute team debrief outside the presentation venue to discuss difficult questions and opportunities for program improvement. A pharmacist preceptor supervised each event and supplied two doses of naloxone to a representative from every housing cooperative.

Three months after each train-the-trainer seminar, attendees received an email request to complete a follow-up assessment online via Qualtrics. The assessment included five multiple-choice questions based on seminar content to assess overdose-related knowledge retention (Appendix) and four statements to assess student pharmacists' attitudes toward harm reduction interventions. The study was completed with an observational cohort design, whereby student pharmacists who participated in community outreach events (intervention) were compared to those who only attended the train-the-trainer seminar (control). Knowledge retention was assessed by calculating the number of correct answers out of five. Harm reduction attitudes were assessed by assigning points to Likert-scale values for each of the four items (strongly agree $=2$, agree $=1$, neutral $=0$, disagree $=-1$, strongly disagree $=-2$ ) and adding scores for all responses to establish a cumulative score (possible range of -8 to +8 ). Statistical analyses were completed via IBM SPSS version 24. Differences between the intervention and control groups in the primary outcomes, cumulative scores for knowledge retention questions and harm reduction attitudes 
statements, were analyzed using the Mann-Whitney $U$ test. Differences in these outcomes by professional year were analyzed using the Kruskall-Wallis $\mathrm{H}$ test. Differences in proportions of correct responses to individual knowledge retention questions by intervention/control group and by professional year were analyzed using the chi-squared test. The University of Texas at Austin Office of Research Support determined this analysis did not require approval by the Institutional Review Board.

\section{Findings}

Of the 365 student pharmacists enrolled in professional years 1-3 at the College of Pharmacy, 116 attended a train-the-trainer seminar and 94 completed the follow-up assessment, resulting in an $81 \%$ response rate. Of the 94 students who completed the follow-up assessment, 36 (38\%) voluntarily participated in at least one community outreach event while $58(62 \%)$ did not participate (Table 1). P3 students made up the smallest proportion of train-the-trainer seminar attendees. Two P3 students participated in more than three outreach events.

Subjects in the intervention group demonstrated superior knowledge retention compared to the control group, though cumulative harm reduction attitudes did not differ between groups (Table 2). Analyses of these primary endpoints were repeated with the exclusion of all P3 students, then with the exclusion of only those P3 students who participated in more than three outreach events, and the results were unchanged. There was significant variation in knowledge retention by professional year with P3 students obtaining higher cumulative median and mean scores than both P1 and P2 students. There was no significant difference in harm reduction attitudes by professional year. Chi-squared tests showed that the intervention group had a significantly higher proportion of correct answers for four of the five knowledge questions.

Harm reduction statement responses were also analyzed individually (Table 3). Subjects in the intervention group exhibited more positive attitudes on the Likert-scale regarding naloxone access for people who use illicit opioids as assessed by Statement 2. Responses to the other statements did not differ significantly between groups. Statement 1 , which assessed attitudes regarding naloxone access for people who use prescription opioids under medical supervision, elicited the most positive response. 
Statement 4, which assessed attitudes regarding legalization of supervised injection facilities, elicited the least positive response. Evaluated individually, the mean and median scores for all harm reduction responses were positive. The majority of subjects indicated support for supervised injection facilities, an extremely progressive harm reduction intervention.

\section{Discussion}

Our hypothesis that participation in community outreach events would result in superior overdoserelated knowledge retention was supported by the significantly higher mean and median scores achieved on the follow-up assessment by the intervention group compared to the control group. This finding suggests our service learning program provided key opportunities for student pharmacists to reinforce knowledge gained in a didactic setting through application in an experiential setting. Superior recall by the intervention group on four of the five knowledge questions indicates participation in community outreach events contributed to increased knowledge in a variety of content areas. Question 3, for which there was no difference in correct response frequency between groups, was clearly flawed as the correct answer could be guessed relatively easily.

Our hypothesis that participation in community outreach events would result in more positive attitudes regarding harm reduction was rejected based on the similar mean and median scores calculated from the Likert-scale harm reduction statement responses in both groups. This finding suggests our service learning program did not provide the depth of exposure and opportunities for reflection that are necessary to change harm reduction attitudes. It is possible that cumulative harm reduction scores were not different between groups because two of the four statements evaluated attitudes regarding harm reduction strategies that were not directly addressed through Operation Naloxone participation. These strategies, syringe service programs and supervised injection facilities, are gaining increased attention as the opioid crisis escalates. Healthcare provider awareness and acceptance will be critical to their implementation. The intervention group did report significantly more positive attitudes toward naloxone access for individuals who use illicit opioids, indicating some attitudes may have been impacted by the program. The overwhelmingly positive attitudes of all subjects toward harm reduction interventions were 
remarkable. When all harm reduction responses were used to calculate a cumulative score, 92 of 94 subjects exhibited harm reduction attitudes that were more positive than negative.

The voluntary participation of 116 student pharmacists, representing one-third of eligible students and distributed relatively evenly across professional years $1-3$, is a notable strength of this small pilot study. The response rate for the follow-up assessment exceeded $81 \%$, which supports the internal validity of the results. However, the small sample size and single-site nature of the study limit external validity. While the inclusion of both knowledge and attitude assessments provides valuable information for educators, generalizability of the results is limited as the assessment instrument was developed by the faculty director and did not undergo validation. The small number of knowledge-related questions and indirect relationship of some harm reduction statements indicates need for meaningful revision of the assessment instrument. Baseline knowledge and attitudes were not collected, so analyses of their change over time could not be conducted. The observational cohort design leaves this study susceptible to sampling bias as subjects self-selected to participate in outreach events. Several subjects participated in more than one community outreach event, but the sample was too small to compare outcomes based on this variable. Future studies that seek to build on this work should minimize sampling bias by collecting baseline assessment data and considering random assignment of subjects to study groups. Assessing the impact of participation in multiple community outreach events could also be helpful in identifying an ideal degree of exposure to affect change.

\section{Summary}

Student pharmacists at The University of Texas at Austin College of Pharmacy demonstrated enthusiasm for community engagement and overdose prevention through voluntary participation in the Operation Naloxone service learning program. Student pharmacists who participated have demonstrated the ability to recall relevant information to educate patients and advocate for harm reduction in their community. Preliminary results indicate program participation improved knowledge-retention and has the capacity to impact directly-related attitudes. Furthermore, the results highlight a progressive mindset regarding harm reduction among student pharmacists. 


\section{Acknowledgments}

The authors wish to thank J. Nile Barnes for collaborating to provide overdose response training, Lori Holleran Steiker for spurring campus overdose prevention efforts, and Mark Kinzly for obtaining naloxone for program distribution.

\section{References}

1. Rudd RA, Seth P, David F, Scholl L. Increases in drug and opioid-involved overdose deaths United States, 2010-2015. MMWR. 2016;65(50-51):1445-52.

2. Lim JK, Bratberg JP, Davis CS, Green TC, Walley AY. Prescribe to prevent: overdose prevention and naloxone rescue kits for prescribers and pharmacists. J Addict Med. 2016;10:300-8.

3. Wheeler E, Jones TS, Gilbert MK, Davidson PJ. Opioid overdose prevention programs providing naloxone to laypersons - United States, 2014. MMWR. 2015;64(23):631-5.

4. Davis C, Carr D. State legal innovations to encourage naloxone dispensing. J Am Pharm Assoc. $2017 ; 57(2): S 180-4$.

5. Accreditation Council for Pharmacy Education. Accreditation Standards and Key Elements for the Professional Program in Pharmacy Leading to the Doctor of Pharmacy Degree ("Standards 2016"). Published February 2015. Available at: https://www.acpeaccredit.org/pdf/Standards2016FINAL.pdf. Accessed May 16, 2018.

6. Accreditation Council for Pharmacy Education. Guidance for the Accreditation Standards and Key Elements for the Professional Program in Pharmacy Leading to the Doctor of Pharmacy Degree (“Guidance for Standards 2016"). Published February 2015. Available at: https://www.acpeaccredit.org/pdf/GuidanceforStandards2016FINAL.pdf. Accessed May 16, 2018.

7. Haack S, Phillips C. Teaching cultural competency through a pharmacy skills and applications course series. Am J Pharm Educ. 2012;76(2): Article 27. 
8. Brown B, Heaton PC, Wall A. A service-learning elective to promote enhanced understanding of civic, cultural, and social issues and health disparities in pharmacy. Am $J$ Pharm Educ. 2007;71(1): Article 09.

9. Kearney KR. Impact of a service-learning course on first-year pharmacy students' learning outcomes. Am J Pharm Educ. 2013;77(2): Article 34.

10. Kearney KR. A service-learning course for first-year pharmacy students. Am J Pharm Educ. 2008;72(4): Article 86.

11. Kearney KR. Students' self-assessment of learning through service-learning. Am J Pharm Educ. 2004;68(1): Article 29.

12. Falter RA, Pignotti-Dumas K, Popish SJ, et al. A service learning program in providing nutrition education to children. Am J Pharm Educ. 2011;75(5): Article 85.

13. Johnson JF. A diabetes camp as the service-learning capstone experience in a diabetes concentration. Am J Pharm Educ. 2007;71(6): Article 119.

14. Kabli N, Liu B, Seifert T, Arnot Ml. Effects of academic service learning in drug misuse and addiction on students' learning preferences and attitudes toward harm reduction. Am J Pharm Educ. 2013;77(3): Article 63.

15. Panther SG, Bray BS, White JR. The implementation of a naloxone rescue program in university students. J Am Pharm Assoc. 2017;57(2):S107-12.

16. Maguire MA, Pavlakos RN, Mahta BH, Schmuhl KK, Beatty SJ. A naloxone and harm reduction educational program across four years of a doctor of pharmacy program. Curr Pharm Teach Learn. 2018;10:72-7.

17. Monteiro K, Dumenco L, Collins S, Bratberg J, MacDonnell C, Jacobson A, Dollase R, George P. An interprofessional education workshop to develop health professional student opioid misuse knowledge, attitudes, and skills. J Am Pharm Assoc. 2017;57:S112-7.

18. Jacobson AN, Bratberg JP, Monk M, Ferrentino J. Retention of student pharmacists' knowledge and skills regarding overdose management with naloxone. Subst Abus. 2018. 
19. Jordan RP, Bratberg J, Congdon HB, Cross LB, Hill LG, et al. The report of the 2016-2017 advocacy standing committee. Am J Pharm Educ. 2017;81(8):S10. 


\section{Appendix: Knowledge Retention Questions}

1. Which of the following is not an opioid?
a. Heroin
b. Oxycodone
c. Cocaine
d. Fentanyl

2. Which of the following statements best describes naloxone?

a. A stimulant that "wakes you up" from an overdose

b. An opioid blocker that kicks opioids off their receptors

c. A weak opioid that has less of an effect on breathing

d. An opioid binder that inactivates opioids in the bloodstream

3. Which of the following is a common symptom of opioid overdose?
a. Breathing very slowly or not at all
b. Blue/grey nails and lips from lack of oxygen
c. Nonresponsive to stimuli, including sternal rub
d. All of the above

4. Which of the following is the most effective method of administering naloxone?
a. Intramuscular (IM)
b. Intranasal (IN)
c. Intravenous (IV)
d. IM/IN/IV are equally effective

5. Which of the following statements about the "Texas Naloxone Law" is FALSE?

a. Pharmacists may dispense naloxone without a prescription if they are working under a "standing order" from a physician.

b. If two friends are using heroin together and one overdoses, the other can call 911 without fear of prosecution.

c. Anyone who prescribes/dispenses/administers naloxone within the confines of the law is protected from liability.

d. Naloxone can be prescribed to a "third-party" (eg, family/friend) rather than the individual at risk for overdose. 
Table 1: Study Groups by Professional Year

\begin{tabular}{lccc}
\hline & $\begin{array}{c}\text { Overall }(\mathbf{N}=94) \\
\text { n (col \%) }\end{array}$ & $\begin{array}{c}\text { Intervention }(\mathbf{N}=\mathbf{3 6}) \\
\text { n (col \%) }\end{array}$ & $\begin{array}{c}\text { Control }(\mathbf{N}=\mathbf{5 8}) \\
\text { n (col \%) }\end{array}$ \\
\hline P1 Students & $35(37.2)$ & $13(36.1)$ & $22(37.9)$ \\
P2 Students & $36(38.3)$ & $12(33.3)$ & $24(41.4)$ \\
P3 Students & $23(24.5)$ & $11(30.6)$ & $12(20.7)$ \\
\hline
\end{tabular}

$\mathrm{P} 1=$ professional year one; $\mathrm{P} 2=$ professional year two; $\mathrm{P} 3=$ professional year three;

Intervention=attended outreach events; $\mathrm{Control}=\mathrm{did}$ not attend outreach events 
Table 2: Primary Outcome Analyses

\begin{tabular}{lcccc}
\hline & Median & Mean & Std Dev & $\boldsymbol{p}$-value \\
\hline Knowledge Retention & & & & \\
Intervention & 5 & 4.69 & 0.58 & $<0.001^{\mathrm{a}}$ \\
Control & 4 & 3.88 & 1.03 & \\
P1 Students & 4 & 4.00 & 0.91 & \\
P2 Students & 4 & 4.00 & 1.10 & $0.001^{\mathrm{b}}$ \\
P3 Students & 5 & 4.78 & 0.52 & \\
\hline Harm Reduction Attitudes & & & & \\
$\quad$ Intervention & 6 & 5.81 & 1.43 & $0.89^{\mathrm{a}}$ \\
Control & 6 & 5.34 & 2.58 & \\
P1 Students & 6 & 5.69 & 2.56 & \\
P2 Students & 6 & 5.31 & 2.25 & $0.58^{\mathrm{b}}$ \\
P3 Students & 6 & 5.61 & 1.53 & \\
\hline
\end{tabular}

${ }^{a}$ Mann-Whitney U test; ${ }^{b}$ Kruskal-Wallis H test

$\mathrm{P} 1=$ professional year one; $\mathrm{P} 2=$ professional year two; $\mathrm{P} 3=$ professional year three;

Intervention=attended outreach events; Control=did not attend outreach events 
Table 3: Harm Reduction Statement Responses

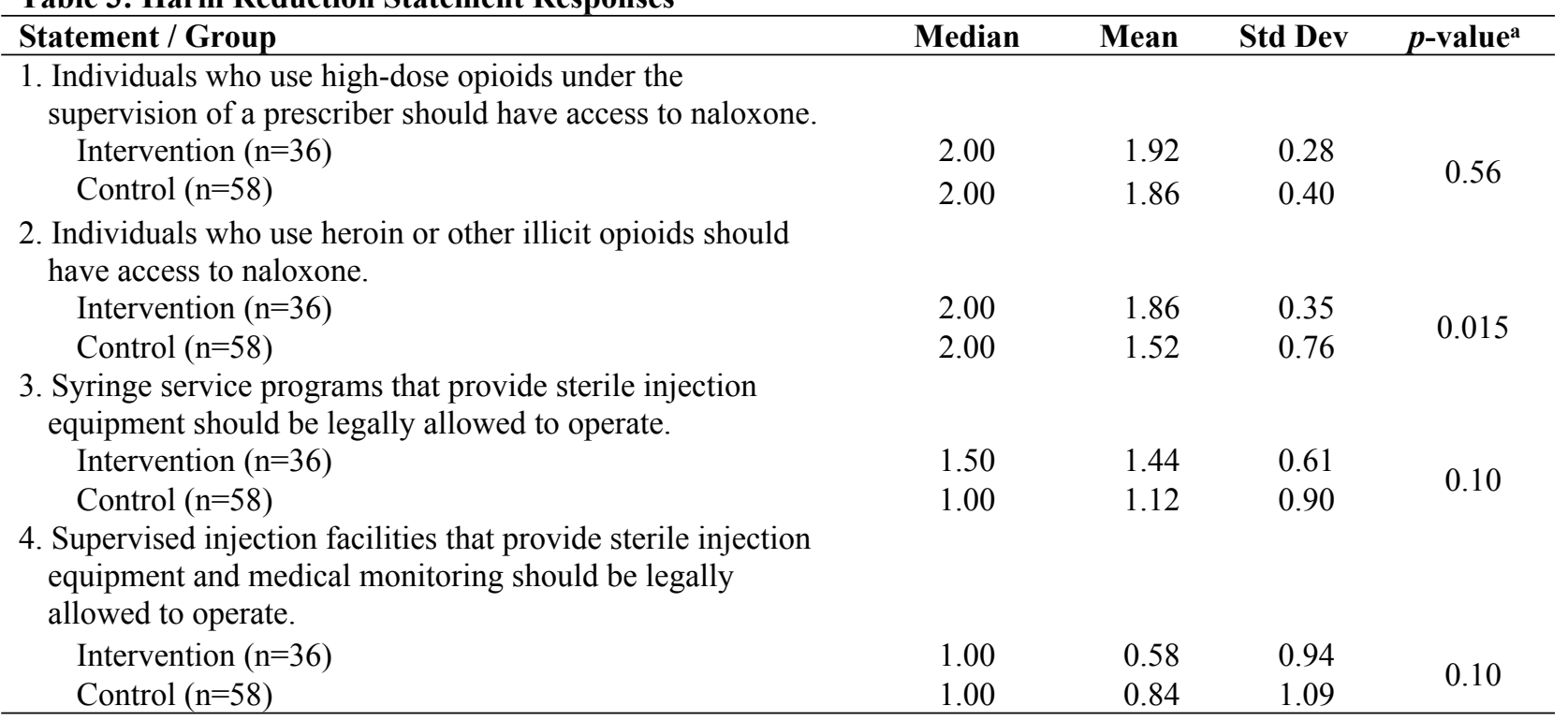

a Mann-Whitney U test

Intervention=attended outreach events; Control=did not attend outreach events 